\title{
Políticas públicas y el uso del internet para el acceso y eficiencia en la presentación de los servicios públicos: caso del Portal de Servicios del Registro Nacional de Costa Rica
}

Public policies and the use of the internet for access and efficiency in the presentation of public services: case of the Portal of Services of the National Registry of Costa Rica

\author{
Georgina Paniagua \\ Universidad de Chile
}

\section{Introducción}

Inmersos en la denominada Sociedad de la Información, es importante preguntarse si ese recurso considerado como el más valioso en el mundo, es o no un tema central en la formulación y la implementación de las políticas públicas de la región latinoamericana. Y más aún, si es un tema que se concibe en términos de igualdad para la ciudadanía y un factor de inclusión social. Las reflexiones que se exponen parten del caso del funcionamiento del Portal de Servicios Digitales del Registro Nacional de Costa Rica. Las políticas públicas involucran un conjunto de acciones $\mathrm{u}$ omisiones en torno a la solución de los problemas de la sociedad; si bien, no es la única alternativa para cerrar las brechas del desarrollo, sí constituyen, de acuerdo con Ruiz y Cadenas (2005), un conjunto de principios, instrumentos y servicios o acciones para cumplir esos principios. Por otra parte, las políticas públicas tienen que ver con el acceso de la población a los bienes y servicios. Desde este punto de vista, es importante asumir una actitud analítica proclive a la mejora continua de la actuación del Estado como agente clave que promueve el bienestar social.

En 1865 se creó el Registro Público de Costa Rica. Dado el desarrollo científico que había alcanzado la gestión de los asuntos registrales, se decide unificar todos los registros existentes; decisión visionaria en materia de política pública de defensa de los derechos en torno al resguardo de la propiedad de los ciudadanos. Es así, como en agosto de 1969, se crea al Registro Nacional como un organismo dependiente del Ministerio de Gobernación. Seis años después, en 1975 se promulga la Ley de Creación del Registro Nacional, la cual deroga la anterior y es la que define el ordenamiento jurídico básico como institución integradora de la actividad registral del país. En noviembre de 1983, el Registro Nacional pasa a formar parte del Ministerio de Justicia, cuando éste último se segrega del Ministerio

*Dirección de correspondencia [Correspondence address]: Georgina Paniagua, Universidad de Chile E-mail: gpaniagu@hotmail.es de Gobernación. Entre 1979 y 1980, se alcanzó un hito importante en la historia registral de Costa Rica, cuando comenzó a funcionar un nuevo sistema de inscripción registral, denominado "Folio Real", basado en el trípode creado por la memoria de un computador, lo cual ubicó, en esa época, al sistema registral costarricense en el más avanzado de la región.

El propósito fundamental del Registro Nacional es registrar en forma eficaz y eficiente, los documentos que se presenten a la Institución, para su inscripción; así como garantizar a los ciudadanos los derechos de propiedad con respecto a terceros. Asimismo, custodiar y suministrar a la colectividad la información los bienes y derechos inscritos o en proceso de inscripción. La visión del Registro Nacional es ser una institución líder en la prestación de servicios registrales y geoespaciales de calidad.

El Registro Nacional, como institución garante de la democracia del país que protege los derechos de propiedad de las personas físicas y jurídicas, es considerada como una organización clave para el desarrollo económico y social del país y es fundamental para la sostenibilidad de relativos bajos niveles de riesgo país, toda vez que garantiza que esos derechos sean una expectativa real que contribuye al desarrollo y bienestar social de la nación.

\section{El portal de servicios}

En la década de los años noventa, el Registro Nacional creó una página informativa en el Internet. Este sitio se conoció como www.registronacional.go.cr; el cual brindó información de consulta a los ciudadanos. El sitio era estático, en cuanto no permitía interacción alguna con los usuarios. El año 2008, se creó un nuevo sitio que cambió el paradigma de las relaciones Estadociudadanía, bajo un nuevo esquema de prestación de servicios, donde el usuario, puede tener acceso a información y servicios en tiempo real, confiable, seguro sobre sus derechos de propiedad. Este Portal de Servicios del Registro Nacional de Costa Rica (en adelante, el Portal), tiene como objetivo "Mo- 
dernizar la prestación de los servicios del Registro Nacional, de manera que los mismos reúnan las mejores condiciones de calidad, seguridad, eficiencia y acceso al público" (Registro Nacional de Costa Rica, 2014). La institución tiene un modelo integral y único de registración de los derechos de propiedad mueble, inmueble (gráfica y literal) y de personas jurídicas. Asimismo, a partir del año 2012, mediante la reforma a la Ley de Creación antes indicada, el Instituto Geográfico Nacional pasó a formar parte de la estructura organizativa del Registro Nacional. Dicho Instituto es la dependencia científica y técnica que funge como rectora de la cartografía del país.

El Proyecto de establecimiento y puesta en marcha del Portal Digital ha conllevado varias etapas y se ha gestado en virtud de una alianza estratégica con el Banco de Costa Rica. Esta alianza, ha permitido realizar importantes inversiones, cuya sostenibilidad financiera ha sido garantizada porque el Portal es económicamente autosostenible, mediante el cobro en línea a los usuarios por la prestación de los servicios.

Las páginas del sitio web son visualizadas por los navegadores web de dispositivos móviles actuales, para hacer uso del Portal, los usuarios se deben registrar de manera gratuita, con lo que pueden adquirir un inventario de servicios para consumir en el acto o varios días después de su adquisición, de acuerdo con sus preferencias. Los principales servicios que brinda el Portal son certificaciones de personas jurídicas, bienes muebles, inmuebles, planos, marcas de ganado e imágenes varias relacionadas con los derechos de propiedad y consultas respecto tanto a las certificaciones como al pago de impuestos y marcas comerciales. Además se puede pedir certificaciones físicas, planos de tamaño real y solicitar placas y reservas de matrícula vehicular. Por último se cuenta con un servicio de Alerta Registral, que brinda mayor seguridad a los usuarios sobre sus derechos de propiedad porque en forma automática avisa sobre solicitudes o trámites de modificación sobre derechos.

Otros esfuerzos de modernización tiene que ver con los servicios que se pondrán a disposición de los usuarios, tales como un nuevo sistema de cálculo y recaudación de ingresos (timbres y aranceles) y un sistema de ventanilla de presentación electrónica de documentos al Registro Nacional, en donde los usuarios podrán ingresar sus solicitudes de inscripción de derechos a través de una ventanilla electrónica.

El Portal permite facilitar la incorporación de usuarios individuales y corporativos (o usuarios intermedios), a través de un módulo de convenios que permite que otras entidades públicas o privadas brinden la información necesaria que posibilite las transacciones jurídicas, económicas, comerciales y otras que requiera la ciudadanía costarricense. A la fecha de redacción de este documento, se tienen suscritos más de cincuenta convenios, los cuales, reflejan ingresos incrementales por ventas de servicios a lo largo y ancho del país.

Los principales beneficios que el Portal genera al país consisten en una disminución sustancial de costos a los usuarios, tanto en tiempo como en dinero, una ampliación del acceso a los servicios y mayor cobertura en las zonas rurales del país. Además ha permitido que el Registro Nacional mejore la calidad de sus servicios y se fortalezca como una institución vital en el desarrollo del país.

\section{Reflexiones desde la óptica de las políticas públicas enfocadas en incrementar la eficiencia de los servicios mediante el uso de internet}

Uno de los principales desafíos de los estados de hoy es la creación de valor público en sus actuaciones, bajo un modelo de Gestión para Resultados de Desarrollo (GpRD). El valor público se refiere al valor creado por el Estado en respuesta a las necesidades y demandas ciudadanas, expresado en bienes, servicios, leyes, etc. (García y García, 2013). El enfoque de GpRD permite analizar la actuación pública en función de la definición de resultados, por tanto, es una estrategia de desarrollo que rescata la integralidad como principio de gestión pública. De allí la importancia, de analizar las políticas públicas del uso del Internet en función de los resultados que se pretenden alcanzar y de esta forma, ubicar las decisiones públicas en distintos contextos de consecución de resultados para obtener como fin el mejoramiento de los niveles del bienestar general de la sociedad.

Las políticas de uso del Internet en la prestación de servicios públicos, para el caso del Registro Nacional se pueden visualizar, desde el punto de vista de los componentes de las políticas públicas, agregándose algunos factores potenciadores de los beneficios de esas políticas que constituyen elementos recomendables a incorporar en los componentes indicados. Los fines se reflejan en dos niveles: los definitorios (establecidos por Ley) o sustantivos y, los que se subordinan a éstos y los apoyan, éstos últimos, son propios de la dinámica de la gestión pública y son transversales (fines intermedios). El 
punto clave es entonces la integración de las políticas desde su diseño, articulando sus instrumentos, servicios y acciones que en última instancia facilitarán su implementación y posterior evaluación.

Se debe considerar también, que no se trata únicamente de ampliar el acceso al Internet, sino que también, se debe incidir en la perspectiva social, apuntalando el cierre de las brechas económicas y sociales, las cuales indican que existen grandes disparidades de desarrollo entre las zonas urbanas, las rurales y las marginales; por tanto, cobra importancia el carácter inclusivo de las políticas públicas.

La formulación de la política pública enfocada en incrementar el acceso de la población al Internet, se configura en un componente transversal de otras políticas públicas de nivel superior. En el caso del Portal de Servicios del Registro Nacional, los objetivos o principios de esa política se han enfocado en coadyuvar al incremento de la competitividad del país, en tanto se ofrece una plataforma digital para el acceso a servicios que facilitan el tráfico mobiliario e inmobiliario del país y en general múltiples actividades de índole comercial, industrial e intelectual del país; bajo los parámetros de la seguridad jurídica y la publicidad registral de los derechos de propiedad. Se convierte en una modalidad de servicio cuyos beneficios son de efecto multiplicador para el impulso de otras políticas de desarrollo que se gestan con una óptica intersectorial, regional e institucional; que abarcan áreas distintas de actividad tales como educación, vivienda, comercio, industria, ambiente, seguridad, planificación, justicia, entre otros.

Ante la necesidad de potenciar los beneficios de la política pública, enfocada en masificar el acceso al Internet, como lo es la digitalización de los servicios del Registro Nacional, es preponderante hacerse algunas interrogantes que permitan analizar aspectos de calidad, considerando su impacto, sus limitaciones y potencialidades. La primera interrogante que surge, es en qué medida el Proyecto del Portal de Servicios ha sido el resultado de una formulación integradora de política pública a nivel gubernamental dirigida a la ampliación del acceso a los servicios públicos digitales.

De acuerdo con la experiencia de este Proyecto, para su impulso ha sido fundamental la existencia de al menos cuatro factores claves: la voluntad de los jerarcas de emprender esfuerzos por lograr la modernización de los servicios que brinda la Institución a los usuarios; la formulación de planes institucionales con componente sistemático de modernización institucional, orientados a resultados; la disponibilidad de recursos humano y materiales; y la alianza estratégica con el Banco de Costa Rica.
Desde el punto de vista de formulación de políticas públicas, este esfuerzo, aun siendo totalmente compatible con la intencionalidad gubernamental de modernizar los servicios, no se visualiza como el resultado de una política emitida a nivel macro del Estado que integre el total accionar gubernamental en materia de ampliación de acceso a la información y a los servicios digitalizados del Estado. La participación del Registro Nacional en ese engranaje de políticas tendientes a lograr una mayor competitividad parece ser más un resultado de la voluntad política del gobierno de turno y la entereza de sus jerarcas en lograrlo. Se percibe la necesidad de que se genere una política articulada a nivel de Gobierno que contemple una vinculación sistemática entre los objetivos estratégicos de gobierno, metas macro, totalmente articulada con otras políticas vinculadas tales como las políticas de cierre de la brecha digital del país, fomento productivo, educación a nivel de las tecnologías de información y comunicación (TIC?s), inversiones en ciencia, tecnología e innovación. Se considera que este debe ser un esfuerzo producto de una visión país de desarrollo y no una prioridad sujeta únicamente a la agenda que deriva de una coyuntura política de corto-mediano plazo.

Es necesario enfocar al Estado en una gestión basada en los resultados esperados para lograr esa visión país. Por consecuencia, se dará una vinculación formal y directa entre los planes nacionales, sectoriales e intersectoriales que logre resultados totalmente congruentes a nivel conceptual y práctico. El accionar institucional para mejorar su provisión de servicios públicos es un esfuerzo loable que parte de un liderazgo institucional que se destaca en el país y cuyos preceptos y prácticas de gestión podrían replicarse en el conglomerado de otros entes públicos que tienen importantes responsabilidades frente a la sociedad en materia de principios, instrumentos, acciones y servicios públicos, elementos que describen a las políticas públicas.

Se han creado consejos liderados por la Presidencia de la República para tratar el tema de la competitividad; en los cuales participan diversos sectores de actividad gubernamental, no obstante, estos instrumentos deberían funcionar de manera permanente, con un dinamismo sostenido en el tiempo. Es necesario forjar una visión país, que se concrete en las iniciativas propias, tales como la formulación de planes nacionales de desarrollo que logren integrar los principales esfuerzos del país en objetivos y metas estratégica de larga data, que "sobrevivan" a los cambios de gobierno.

Otra pregunta que debe realizarse, es cuáles factores se consideran decisivos para potenciar los beneficios sociales de la política de ampliar el acceso a 
los servicios públicos a través del Internet. El considerar la política pública del uso del Internet en la población, necesariamente conlleva a analizar en general, cómo se han concebido las políticas de cierre de la brecha digital del país, dado que se trata de que cada vez más personas puedan tener acceso al Internet, y que además tengan la educación indispensable para saber su uso, potencialidades y alcances de sus contenidos, incluso participar en su generación.

El término brecha en este caso se refiere al nivel de acceso que se tiene a las TIC?s. Estas, entendidas como aquellas tecnologías de redes de telecomunicaciones e informática (teléfono, radio, televisión, Internet, computadoras, etc.) que influyen en la vida. No toda la población tiene acceso a las TIC?s y esto genera tales brechas, las cuales limitan a su vez, el desarrollo de capacidades de lograr emprendimientos importantes para superar limitados estadios de desarrollo. Esto incluye obviamente la limitación de esas comunidades al acceso a los servicios públicos que se han puesto a disposición a través del Internet, como en el caso expuesto.

A nivel mundial, de acuerdo con la publicación disponible a la fecha de redacción de este artículo, respecto del ranking efectuada por el Sitio Internet Word Stats, 2011, entre los 50 países medidos por su penetración al Internet, los tres países en mejor posición son Islandia, con un $97.8 \%$, Noruega y Suecia con $97.2 \%$ y $92.9 \%$ respectivamente. De América Latina únicamente aparece Argentina con un $67 \%$ de penetración y ocupando el lugar 46 de 50. Datos más recientes de la misma fuente, de diciembre del 2013, indican para este país un $75 \%$ de penetración. Existen grandes desafíos en la región para lograr una mayor y mejor penetración del Internet que beneficie a toda la población. Este aspecto se encuentra presente en cifras más recientes, donde es posible identificar la vigencia de tales retos.

América Latina y el Caribe, representan a finales del 2013 únicamente un 10,6\% del total de usuarios de Internet del mundo. Y aun cuando los dispositivos móviles contribuyen a la disminución de la brecha digital, a la vez que se incrementa el número de usuarios en la región, se indica que la penetración de Internet sigue siendo baja porque persisten problemas de infraestructura que garantice un acceso real y de aprovechamiento de contenidos en cobertura suficiente en la población. A nivel de América Latina, según los datos disponibles en el 2011, Costa Rica ocupaba el quinto lugar en el ranking. Sobre esa posición se encontraban Argentina, Chile, Uruguay y Panamá. A finales del 2013, se mantiene Argentina en la primera posición, luego le siguen en ese orden, Chile, Colombia, República Dominicana,
Uruguay, Brasil y Costa Rica, pasando este último país a una posición de sétimo lugar.

No obstante, datos más recientes indican que, en el caso de Costa Rica, se anota un alto crecimiento de usuarios de Internet de banda ancha móvil, más que todo por el predominio incremental de las líneas celulares, las cuales representan un $151 \%$, la más alta de Centro América. Los usuarios de Internet se han incrementado (del 2010 al 2013) en un $485 \%$. Por otra parte, las suscripciones a Internet fijo crecieron en un $25 \%$ en ese periodo y al 2014, el $37 \%$ de las viviendas tienen acceso a una conexión de Internet fijo.

Por otra parte, la conectividad, que permite medir la capacidad que tienen los países para utilizar las TIC?s para su desarrollo y bienestar social, si bien es cierto se ha incrementado se mantiene como un desafío importante para cerrar la brecha digital.

De acuerdo con Pablo Bello (2014), Secretario General de la Asociación Iberoamericana de Centros de Investigación y Empresas de Telecomunicaciones (Ahciet), se debe reconocer que aún con esos desafíos, América Latina ha progresado de manera sustancial en los últimos años en materia de telecomunicaciones y acceso a nuevas tecnologías. Esto por cuanto, se tienen más familias conectadas, las velocidades promedio se han estado incrementando y los precios reales han bajado. Atribuye este progreso a las inversiones del sector empresarial, la competencia de mercados y las políticas públicas que en general, han favorecido estos avances.

Por otra parte, la educación en las TIC?s es otro importante desafío que en forma conexa está ligada a converger para el cierre de las brechas del desarrollo económico y social del país, para lograr un desarrollo más justo y equitativo. Políticas que incentiven el desarrollo local de contenidos y aplicaciones es parte de los desafíos a asumir. Se considera importante, que, aun cuando el Ministerio de Educación no forma parte, según el decreto antes indicado, del recientemente creado Consejo Presidencial de Competitividad, sea partícipe de este órgano, por cuanto, la educación desempeña un rol preponderante en los temas de competitividad del país.

Una última pregunta que debe realizarse es qué aspectos de mejora se visualizan para lograr una mayor articulación de las políticas públicas que buscan elevar la competitividad e innovación del país.

Considerando que las políticas públicas tienen tres componentes básicos: los principios, los instrumentos y los servicios que ofrecen a la colectividad, es factible analizar que esos componentes deben fortalecerse mediante la aplicación de un enfoque de 
Gestión de Resultados para el Desarrollo, de suerte que permita aglutinar todos los esfuerzos existentes de una manera articulada y coherente para lograr una mayor eficiencia y eficacia en la provisión de los servicios públicos. La formulación de las políticas públicas en el ámbito de dar mayor acceso a los ciudadanos a los servicios públicos mediante la implementación de esquemas de gobierno electrónico debe ser parte de una visión integrada que contemple la solución de problemas que trasciendan los esfuerzos aislados por lograr estos objetivos.

Esta visión integrada debe contemplar la solución de algunos problemas como el cierre de la brecha digital, entendida como la distancia existente entre las personas que utilizan la Tecnologías de la Información en su vida diaria y las qué no. Además, debe considerar la simplificación de trámites a la ciudadanía; la atracción de la inversión extranjera en el país, la protección de los derechos de propiedad intelectual; una mayor inversión en investigación, desarrollo e innovación; y formular políticas integradas en torno a gobierno electrónico, que permitan avanzar aún más en esta materia en el país.

\section{Conclusiones}

Como resultado de estas reflexiones, planteadas como interrogantes, se concluye que es indispensable la formulación de políticas públicas integradas en relación con el uso de las tecnologías de la información y comunicación, particularmente, el uso del Internet para la provisión de servicios públicos, como es el caso del Portal de Servicios del Registro Nacional. Se comentó que esa integración de políticas se podría lograr en gran parte mediante la aplicación del enfoque de la Gestión por Resultados para el Desarrollo porque permitiría en Costa Rica, articular todos los esfuerzos aislados para fomentar un mayor acceso de los ciudadanos a los servicios públicos, principalmente, aquellos que facilitan directamente el desarrollo económico y social del país. En síntesis, lograr una mayor generación de valor público, a partir de un accionar planificado, bajo un esquema que mantenga total claridad sobre los principios, los instrumentos y los servicios o acciones de estas políticas públicas inclusivas tan necesarias para lograr un mayor nivel de bienestar social a la población.

La digitalización de la sociedad debe entenderse como un factor de desarrollo, pero también de equidad y que por ende, requiere de políticas públicas en materia de TIC?s vinculadas con el cierre de brechas socioeconómicas. Se requiere una visión de país de largo plazo con planes articulados bajo el enfoque de una Gestión para Resultados del Desarrollo que integre los sectores de actividad estatal y con una activa participación de las empresas y sociedad civil en general.

\section{Referencias}

García, L. R. y García, M. (2013). La Gestión por Resultados en el Desarrollo: Avances y Desafíos en América Latina y el Caribe. Material de curso "Diseño de Políticas y Programas Públicos e Indicadores de Desempeño". Asocio Consultores y Universidad de Chile.

Ruiz, D. y Cadenas, E. (2005). Â¿Qué es la política pública? Revista Jurídica, $\mathrm{V}(18)$. 